“C 2019 IEEE. Personal use of this material is permitted. Permission from IEEE must be obtained for all other uses, in any current or future media, including reprinting/republishing this material for advertising or promotional purposes, creating new collective works, for resale or redistribution to servers or lists, or reuse of any copyrighted component of this work in other works." 


\title{
Wideband Filtering Out-of-Phase Power Dividers Using Slotline Resonators and Microstrip-to-Slotline Transitions
}

\author{
He Zhu ${ }^{\# 1}$, Jing-Yu Lin ${ }^{\#}$, Y. Jay Guo \\ ${ }^{\#}$ School of Electrical and Data Engineering, University of Technology Sydney, Australia \\ ${ }^{1}$ he.zhu@uts.edu.au
}

\begin{abstract}
Two wideband filtering out-of-phase power dividers are designed and analyzed. The initial design is composed of a quarter-wavelength slotline resonator and two microstrip-toslotline transitions. Three transmission poles are produced to form the passband. Excellent matching and isolation are achieved using extra matching network. Based on the initial design, a pair of stepped-impedance open stubs are shunted at the output ports to improve the passband selectivity. The designs have been verified through experiment. The tested results show that the proposed devices have achieved $60 \%$ operating bandwidth, $1.8 \mathrm{~dB}$ insertion loss, $1.2^{\circ}$ phase error and excellent in-band matching and isolation.
\end{abstract}

Keywords-Filtering, out-of-phase, power dividers, slotline, wideband.

\section{INTRODUCTION}

Power dividers are critical microwave components in various kinds of wireless communication systems, such as power amplifiers, mixers, reflectometers, and feeding networks for antenna arrays [1]. In terms of the phase between output signals, power dividers (PD) can be categorized into two types: in-phase type [2]-[4] and out-of-phase one [5]-[8]. The in-phase power dividers have been widely investigated; however, the out-of-phase ones are usually ignored due to the inverted phase between two output ports. Dual-band and wideband performance were obtained in [5] and [6] using doubled-sided parallel striplines and microstrip to slotline transitions. A further design was then proposed in [7] to achieve ultra-wideband by adding extra isolating network at output ports. Filtering responses were introduced in an out-ofphase power divider design in [8] based on multiple broadside coupled lines in low-temperature co-fire ceramic technology.

Although a filtering out-of-phase PD was successfully realized in [8], the design is suffered from narrow bandwidth and complicated fabrication process. This paper mainly focuses on developing a new type of planar filtering out-ofphase power divider with wide operating band. Similar to [5] and [7], this work also adopts the slotline resonator and microstrip-to-slotline transitions due to their multiple resonances and phase inverting property. The design is built using microstrip lines on the printed circuit board, and the related geometries are derived from equivalent circuit analysis. Finally, after fabricating and testing two prototypes, the design approach is successfully verified through experiment.

\section{DESIGN AND ANALYSIS}

\section{A. Wideband Out-of-phase Power Dividers}

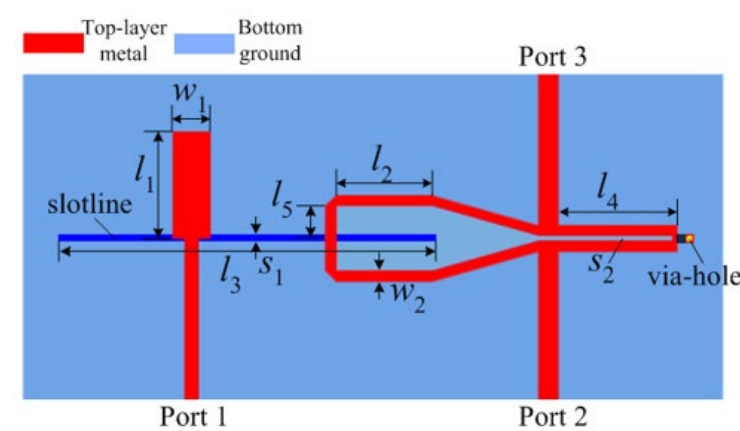

Fig. 1. Layout of the initial design.

Fig. 1 shows the configuration of the presented out-of-phase power divider (PD) with a wide operating and isolation band. The input port 1 is connected with a microstrip-to-slotline transition which can coupled the signals from microstrip lines to the slotline, while a slotline-to-microstrip transition is used to split the input power into two paths. Compared with the design presented in [5], this design uses two extra transmission lines and a lumped resistor to achieve wide isolation bandwidth, considering the fact that the isolation of the design in [5] is only $-8 \mathrm{~dB}$.

The design can be modelled using a transmission-line equivalent circuit, as given in Fig. 2. The microstrip-toslotline transition can be modelled using a transformer with turns ratio of $\mathrm{n}_{\mathrm{s}}$. The microstrip line open stub and slotline short-ended stub are $\left(Y_{s t}, \theta_{s t}\right)$ and $\left(Y_{s}, \theta_{s}\right)$, and the slotline resonator is $\left(Y_{s l}, \theta_{s l}\right)$. The output port 2 and port 3 are connected with two transmission lines $\left(Y_{1}, \theta_{1}\right)$ and $\left(Y_{2}, \theta_{2}\right)$, and a resistor $R$ is grounded at the end. The microstrip line open stub and slotline short-ended stub are required to be quarter-wavelength at the centre frequency, while $\theta_{s l}, \theta_{1}$ and $\theta_{2}$ are set to be $90^{\circ}, 180^{\circ}$ and $90^{\circ}$, respectively.

To investigate the performance of the three-port network, even- and odd-mode analysis method is adopted to simplify the circuit. Fig. 3(a) and (b) show the even- and odd-mode transmission-line equivalent circuit, respectively. For the even-mode case, the centreline of the slotline can be regarded as a perfect magnetic-wall, which will prevent the signals propagating from the slotline to the microstrip lines. Consequently, in Fig. 2, the second transformer will be opencircuit in the middle point, which lead to completely open circuit at the end point of the transmission line of $\left(Y_{1}, \theta_{1}\right)$. The input admittance of the even-mode circuit can be expressed as:

$$
Y_{\text {in,even }}=j Y_{1} \tan \theta_{1}+Y_{2} \frac{Y_{2}+j \frac{1}{2 R} \tan \theta_{2}}{\frac{1}{2 R}+j Y_{2} \tan \theta_{2}}
$$






Fig. 2. Equivalent circuit of the initial design.



(a)

(b)

Fig. 3. Even- and odd-mode equivalent circuit: (a) even-mode; (b) odd-mode.

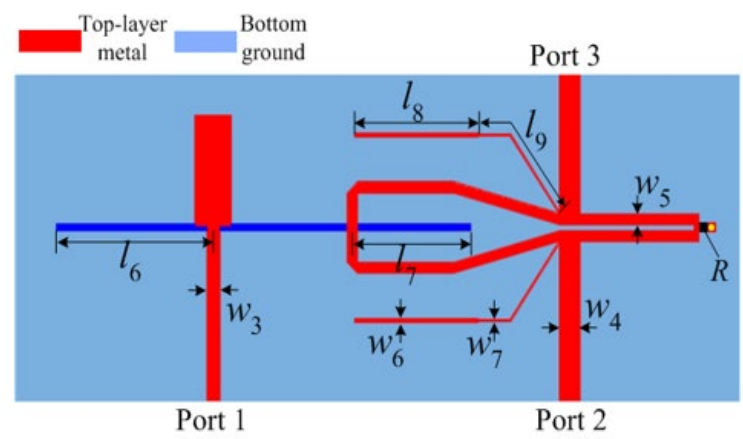

Fig. 4. Layout of the wideband filtering PD with TZs.

To match the output port to $Y_{o}$ and provide ideal isolation at center frequency $f_{o}$, it is required to have $Y_{o}=Y_{2}^{2} \cdot 2 R$. Therefore, the value of $R$ should be chosen as $Z_{2}^{2} / 2 Z_{o}$. In this design, $Z_{2}$ is selected as $\sqrt{2} Z_{o}$ and thus $R$ is equal to $Z_{o}$. For the odd-mode circuit, the input admittance can be written as:

$$
Y_{i n, o d d}=-j Y_{2} \cot \theta_{2}+Y_{1} \frac{Y_{1}+j Y_{l 1} \tan \theta_{1}}{Y_{l 1}+j Y_{1} \tan \theta_{1}}
$$

where

$$
\begin{gathered}
Y_{l 1}=-j Y_{S S} \cot \theta_{S}+Y_{S l} \frac{\left(\frac{Y_{O}}{2}-j Y_{S S} \cot \theta_{S}\right)+j Y_{S l} \tan \theta_{1}}{Y_{S l}+j\left(\frac{Y_{O}}{2}-j Y_{S S} \cot \theta_{S}\right) \tan \theta_{1}} \\
Y_{S S}=\left(\frac{2}{n_{S}}\right)^{2} \cdot Y_{S}
\end{gathered}
$$

Considering (2)-(4), one can find that condition for achieving perfect matching at both input port and output port is $Y_{o}^{2}=2 Y_{s l}^{2}$. In this case, the impedance of the slotline should be chosen as $Z_{s l}=\sqrt{2} Z_{o}$. Moreover, other parameters such as $Y_{1}$ and $Y_{S}$ should be determined based on the bandwidth requirement.

\section{B. Wideband PD with Transmission Zeros}

To further sharpen the selectivity of the filtering PD's passband, it is desired to introduced more transmission zeros (TZ) at two edges of the passband. To that end, a pair of stepped-impedance open-circuit stubs is shunted at feeding point of output ports, as shown in Fig. 4. Fig. 5 displays the equivalent circuit of the wideband out-of-phase PD with transmission zeros, where the SIOSs are taped at port 2 and

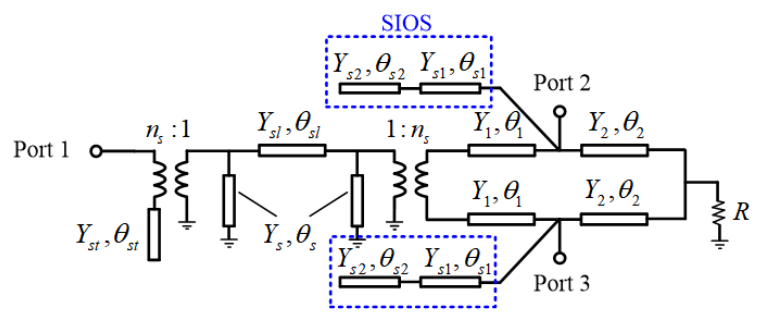

Fig. 5. Equivalent circuit of the wideband filtering PD with TZs.

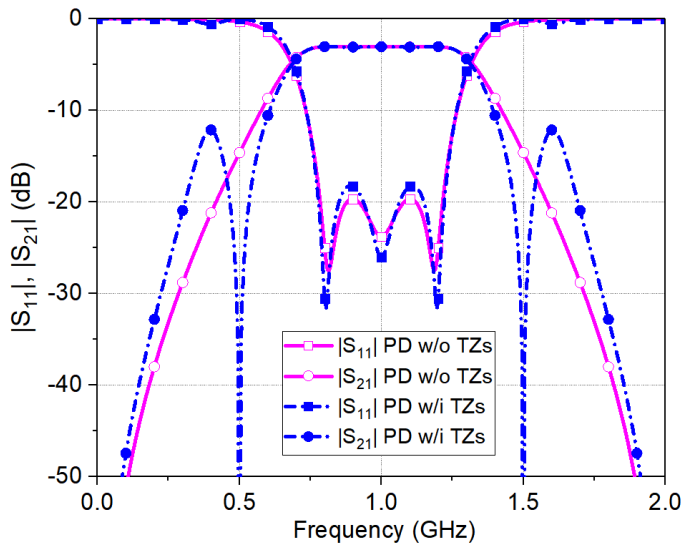

(a)

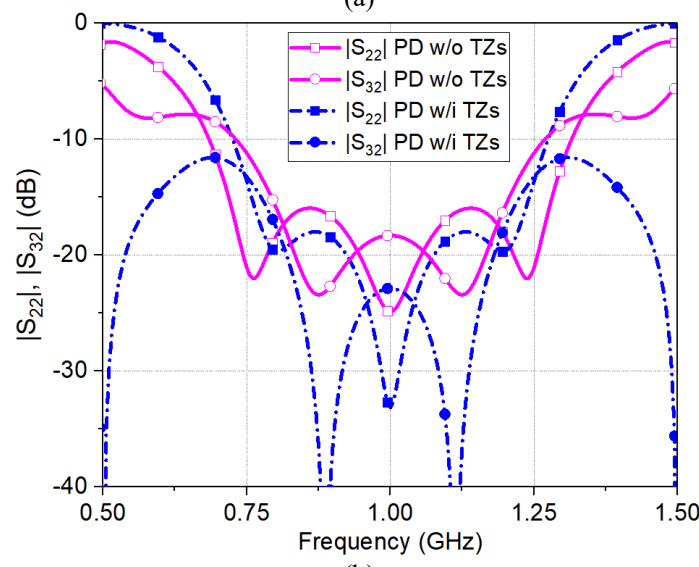

(b)

Fig. 6. S-parameters of two PDs based on the equivalent circuits: (a) $\left|\mathrm{S}_{11}\right|$ and $\left|\mathrm{S}_{21}\right| ;\left(\right.$ b) ) $\left|\mathrm{S}_{22}\right|$ and $\left|\mathrm{S}_{32}\right|$.

port 3. The lengths of each stage of the SIOS $\left(\theta_{s 1}\right.$ and $\left.\theta_{s 2}\right)$ are fixed at quarter-wavelength at $f_{o}$. The shunted SIOSs can introduce a pair of TZs and also more resonant poles to the transmission coefficient of the PD. The input impedance of the SIOS can be expressed as:

$$
Y_{\text {sios }}=Y_{s 1} \frac{j Y_{s 2} \tan \theta_{s 2}+j Y_{s 1} \tan \theta_{s 1}}{Y_{s 1}-Y_{s 2} \tan \theta_{s 1} \tan \theta_{s 2}}
$$

To yield transmission zeros at certain frequencies, it is required to have $Y_{\text {sios }}=\infty$. If we define $Y_{s 1} / Y_{s 2}=K$, it is easy to have $K-\tan ^{2} \theta_{t z}=0$. Solving this equation, one can find the positions of the transmission zeros $f_{t z}$ are located at:

and

$$
\begin{aligned}
& f_{t z 1}=\frac{2}{\pi}(\arctan \sqrt{K}) f_{o}, \\
& f_{t z 2}=\left(2-\frac{2}{\pi} \arctan \sqrt{K}\right) f_{o}
\end{aligned}
$$




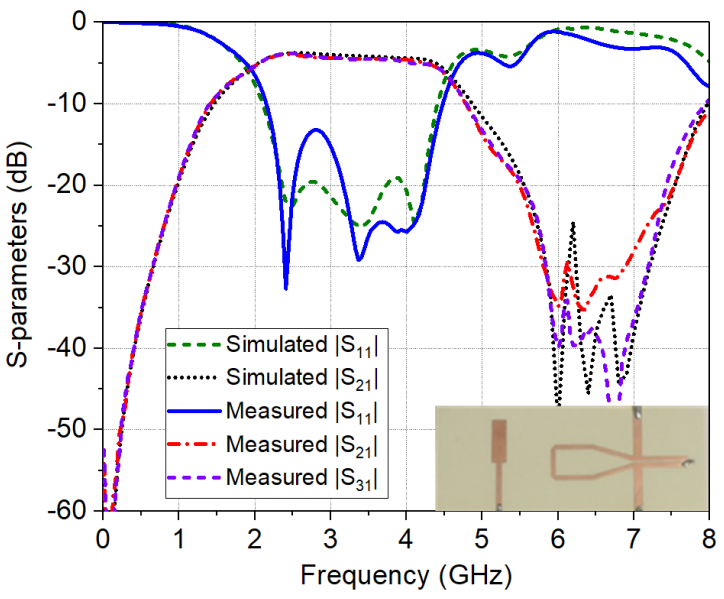

(a)

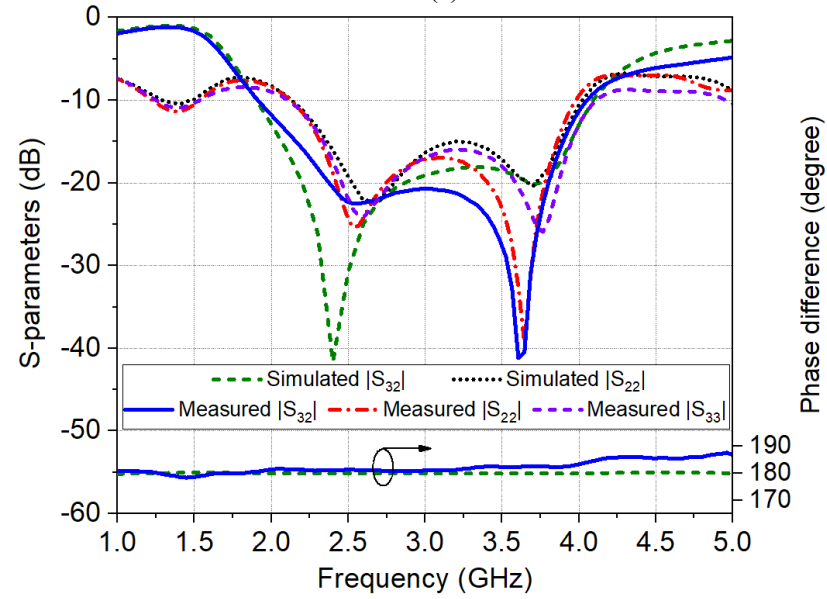

(b)

Fig. 7. Simulated and measured results are the wideband PD without TZs: (a) $\left|\mathrm{S}_{11}\right|,\left|\mathrm{S}_{21}\right|$ and $\left|\mathrm{S}_{31}\right| ;$ (b) $\left|\mathrm{S}_{22}\right|,\left|\mathrm{S}_{33}\right|,\left|\mathrm{S}_{32}\right|$ and phase difference.

It is obvious that $f_{t z 1}$ and $f_{t z 2}$ are located at the upper and lower edge of the passband, which help to improve the selectivity of the filtering band. To depict the performance of two different PDs, the S-parameters are plotted in Fig. 6. It is seen that compared with the first wideband filtering PD, the second design have similar in-band characteristics but feature two TZs and a sharper skirt selectivity. Both two devices have ideal output matching and perfect matching across the same band range with the filtering passband. The phase difference in two designs is constant at $180^{\circ}$ due to the intrinsic out-ofphase property of the microstrip-to-slotline transition.

\section{EXPERIMENT AND RESULTS}

To verify the design, simulations have been conducted on the models displayed in Figs. 1 and 4 using EM simulator ANSYS Electronics Desktop version 17.2. The substrate used in both designs is Rogers RO4350B with dielectric constant of 3.48 and thickness of $0.76 \mathrm{~mm}$. The relevant dimensions in these two figures are listed as: $l_{1}=13, l_{2}=11.7, l_{3}=48.6$, $l_{4}=14.5, l_{5}=4, l_{6}=15.8, l_{7}=15.8, l_{8}=15.3, l_{9}=15.7$, $w_{1}=4.5, w_{2}=1.3, w_{3}=1.6, w_{4}=1.5, w_{5}=1.6, s_{1}=$ $0.12, s_{2}=0.64$, all in millimeter. Besides, the isolation resistor $R$ is chosen as $50 \Omega$.

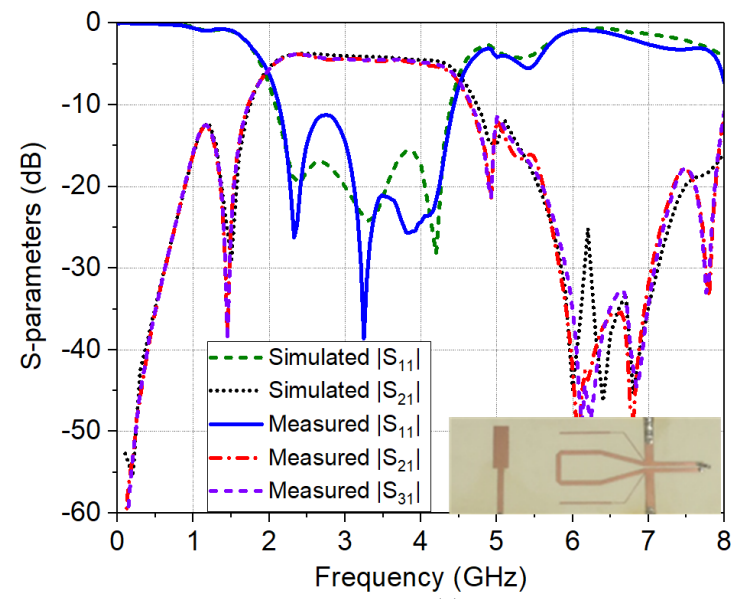

(a)

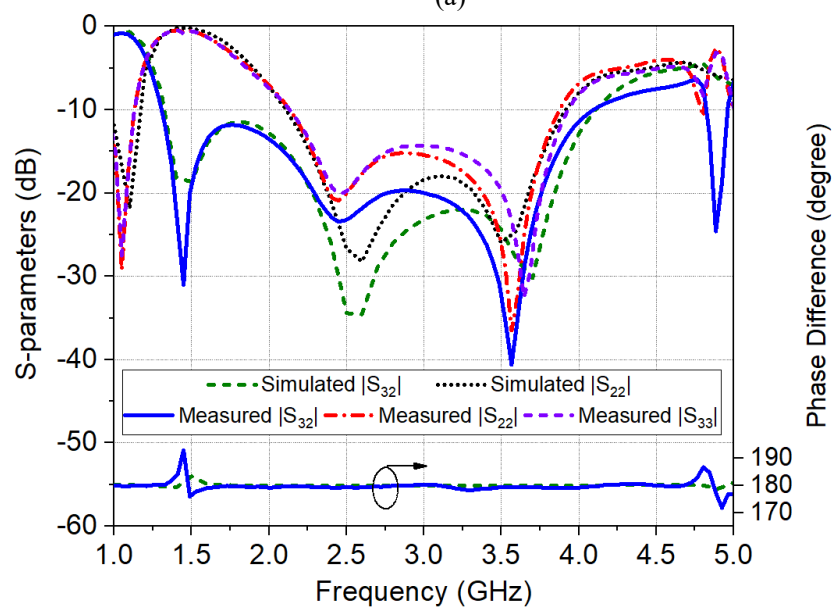

(b)

Fig. 8. Simulated and measured results are the wideband PD with TZs: (a) $\left|S_{11}\right|,\left|S_{21}\right|$ and $\left|S_{31}\right| ;\left(\right.$ b) $\left|S_{22}\right|,\left|S_{33}\right|,\left|S_{32}\right|$ and phase difference.

Finally, two prototypes have been fabricated and tested. Figs. 7 and 8 show the comparison between the measured results with the simulated ones, where a photograph of two prototypes is also embedded. The performance of the first wideband power divider is displayed in Fig. 7, where a flat band is formed with three poles. Considering the matching and isolation at the outputs, the operating band range is from 2.15 $\mathrm{GHz}$ to $3.98 \mathrm{GHz}$ (fraction bandwidth is $59.7 \%$ ). For the power divider with TZs, the band range is from $2.1 \mathrm{GHz}$ to 3.9 $\mathrm{GHz}$ (fraction bandwidth is $60 \%$ ). In both cases, the in-band insertion loss is around $1.8 \mathrm{~dB}$, and the phase difference between port 2 and port 3 is around $3^{\circ}$ and $1.2^{\circ}$. Two transmission zeros are located at $1.46 \mathrm{GHz}$ and $4.98 \mathrm{GHz}$, respectively. In both designs, the output matching $\left(\left|\mathrm{S}_{22}\right|\right.$ and $\left.\left|\mathrm{S}_{33}\right|\right)$ is less than $-15 \mathrm{~dB}$, and the measured isolation $\left(\left|\mathrm{S}_{32}\right|\right)$ is less than $-20 \mathrm{~dB}$. Compared with previously presented works, this work has achieved wideband out-of-phase power dividing performance with filtering response and excellent matching and isolation.

\section{CONCLUSION}

Wideband filtering out-of-phase power dividers have been investigated. The design is developed on a single substrate using microstrip lines and slotlines. Excellent output port 
matching and isolation are realized due to the matching circuit, which guarantees a wide operating bandwidth. The proposed devices integrated the functions of filtering and power splitting, which are very useful in the miniaturization of communication systems.

\section{REFERENCES}

[1] D. Pozar, Microwave Engineering, 3rd ed. New York: Wiley, 2005.

[2] S. W. Wong and L. Zhu, "Ultra-wideband power divider with good inband splitting and isolation performances," IEEE Microw. Wireless Compon. Lett., vol. 18, no. 8, pp. 518-520, Aug. 2008.

[3] S. S. Gao, S. Sun, and S. Q. Xiao, "A novel wideband bandpass power divider with harmonic-suppressed ring resonator," IEEE Microw. Wireless Compon. Lett., vol. 23, no. 3, pp. 119-121, Mar. 2013.

[4] L. Guo, A. Abbosh, and H. Zhu, "Ultra-wideband in-phase power divider using stepped-impedance three-line coupled structure and microstrip- to-slotline transitions," Electron. Lett., vol. 50, no. 5, pp. 383-384, Feb. 2014.

[5] G. L. Dai, X.-C. Wei, E. P. Li, and M.-Y. Xia, "Novel dual-band outof-phase power divider with high power-handling capability," IEEE Trans. Microw. Theory Tech., vol. 60, no. 8, pp. 2403-2409, Aug. 2012.

[6] M. E. Bialkowski and A. M. Abbosh, "Design of a compact UWB outof-phase power divider," IEEE Microw. Wireless Compon. Lett., vol. 17, no. 4, pp. 289-291, Apr. 2007.

[7] U. T. Ahmed and A. Abbosh, "Wideband out-of-phase power divider using tightly coupled lines and microstrip to slotline transitions", Electron. Lett., vol. 52, no. 2, pp. 126-128, 2016.

[8] X. Y. Zhang, X.-F. Liu, Y.-C. Li, W.-L. Zhan, Q. Y. Lu, and J.-X. Chen, "LTCC out-of-phase filtering power divider based on multiple broadside coupled lines," IEEE Trans. Compon., Packag., Manuf. Technol., vol. 7, no. 5, pp. 777-785, May 2017. 